\title{
Managing solitary choroidal tuberculoma in an immunocompetent patient
}

\begin{abstract}
Aim: We describe a case of young immunocompetent 18-year-old female patient who presented with sudden painless diminution of vision in left eye with vision of 20/100 on Snellen's chart. Retinal evaluation showed a massive solitary choroidal granuloma. A positive Mantoux test $(20$ X20 mm) was noted. The patient was started on anti-tubercular therapy and oral steroids and showed resolution of the granuloma with subretinal fluid at macula. After 3 months of oral therapy, the persistent subretinal fluid was managed with intravitreal injection of triamcinolone acetonide $(2 \mathrm{mg})$. This case highlights favorable resolution of granuloma with oral therapy and a safer efficacy of triamcinolone acetonide in treating subretinal fluid due to choroidal tuberculoma with good visual outcome.
\end{abstract}

Introduction: Choroidal tuberculoma is a rare ocular form of tuberculosis (TB) and is a diagnostic and a therapeutic challenge, especially when occurring without other manifestations of the disease. ${ }^{1,2}$ Involvement of the choroid occurs by direct infection or indirect immune-mediated hypersensitivity and is the commonest clinical manifestation of intraocular TB. ${ }^{3}$ The presentation varies from a solitary choroidal granuloma (tuberculoma) to multiple choroidal tubercles, a subretinal abscess or choroiditis. We present a case of an immunocompetent patient, who presents with massive choroidal granuloma and responded to oral antitubercular and steroid therapeutic regimen. Visual acuity improved with intravitreal triamcinolone acetonide injection which resulted in resolution of subretinal fluid after 3 months.

Keywords: choroidal tuberculoma, Triamcinolone acetonide, immunocompetent
Volume 10 Issue 5 - 2020

\author{
Abhinav Dhami,' Nimrata bajaj Dhami, ${ }^{2}$ \\ Ravinder kaur Malhi, ${ }^{3}$ Gobinder Singh \\ Dhami $^{4}$ \\ 'Consultant Vitreo-retina, Dhami eye care hospital, India \\ ${ }^{2}$ Consultant Cataract, refractive and Cornea, Dhami eye care \\ hospital, India \\ ${ }^{3}$ Head Vitreo - Retina department, Dhami eye care hospital, India \\ ${ }^{4}$ Medical director, Dhami eye care hospital, India
}

Correspondence: Abhinav Dhami, Consultant Vitreo-retina, Dhami eye care hospital, 82-b kitchlu nagar, Ludhiana, Punjab, India, Email drabhinavdhami@gmail.com

Received: September 05, 2020 | Published: October 20, 2020

\section{Case report}

An 18-year-old female, presented with sudden diminution of vision since 7 days in left eye. The best corrected visual acuity in right eye was 20/20 and left eye was 20/100 on Snellen's chart. On examination anterior chamber and vitreous was quiet, disc was within normal limits with a yellowish white subretinal mass with small hemorrhage on the surface along the inferior arcade up to mid periphery measuring 12DD(disc diameter) (Figure1) with presence of SRF involving the macula and the inferior quadrant was noted. The b-scan showed mild vitreous echoes with a dome-shaped choroidal lesion with a dimension of $6.82 \times 14.50 \mathrm{~mm}$, with no surface reflectivity irregular low-to-medium internal reflectivity with the presence of an anechoic space in the mass, suggestive of an inflammatory etiology (Figure2). Complete physical examination and medical evaluation findings, including a chest $\mathrm{x}$-ray film, were normal. Results of a blood uveitis workup (serum angiotensin converting enzyme, serum lysozyme, Venereal Disease Research Laboratory titer, Toxoplasma titer, Toxocara titer) were negative. A purified protein derivative skin test was highly positive (induration, 20X20 $\mathrm{mm}$ ). Optical coherence tomography showed subretinal fluid (SRF) at macula (Figure 3). We made the clinical diagnosis of solitary choroidal tuberculoma in an immunocompetent patient in whom routine investigations failed to identify systemic tuberculosis infection. The patient was started on isoniazid, rifampin, and ethambutol for a 6-month period. Under supervision of a physician, systemic steroids $(1 \mathrm{mg} / \mathrm{kg} /$ day $)$ in tapering doses were given. The choroidal granuloma subsequently decreased in size (Figure $1 \mathrm{~A}-\mathrm{C}$ )(Figure $2 \mathrm{~B}$ ) over the following 3 months to form a flat chorioretinal scar. After 3 months of oral ATT and steroids, no improvement in visual acuity (20/100) was noted due to persistent SRF at macula (Figure 3B), intravitreal triamcinolone acetonide 2 milligram was planned and resulted in complete resolution of the SRF at macula (Figure 3C) with shrinkage of the choroidal lesion at 4 month (Figure 1D,E).The final visual acuity was 20/20 and was maintained till 1 year follow up.

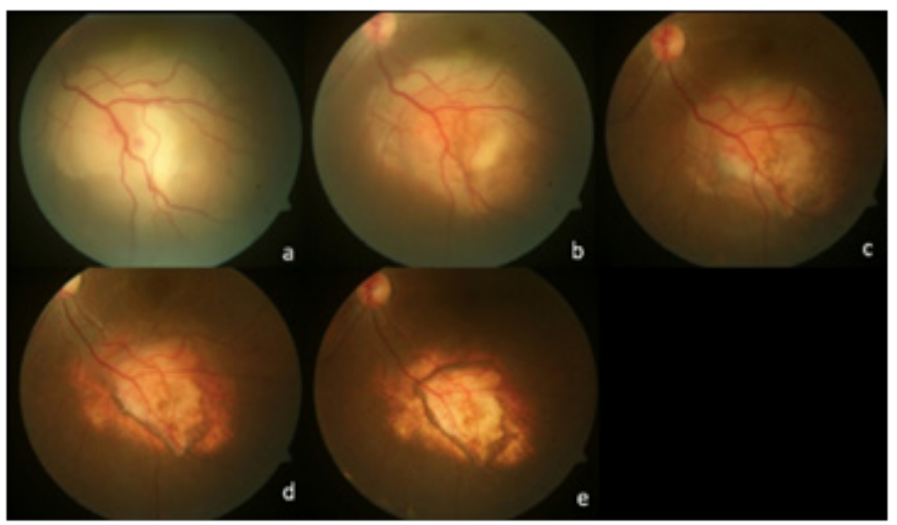

Figure I (A) Shows yellowish white subretinal mass along the inferior arcade up to mid periphery. (B) At I month post starting oral ATT and steroids shows reduction in the size of granuloma. (C) At $3^{\text {rd }}$ month shows further reduction in the size of granuloma with the inferior margin shows signs of atrophy and pigmentary changes. (D) I week post intravitreal triamcinolone acetonide shows gross atrophy at the margins with marked shrinkage in the center. $(E)$ Shows complete shrinkage of the granuloma with central scarring $\left(6^{\text {th }}\right.$ month). 


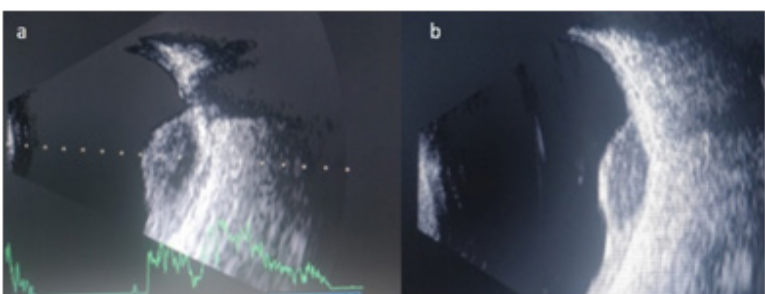

Figure 2 (A) b-scan ultrasonography showed mild vitreous echoes with a dome-shaped choroidal lesion with a dimension of $6.82 \times 14.50 \times 5.5 \mathrm{~mm}$, with irregular low-to-medium internal reflectivity with the presence of an anechoic space in the mass. (B) b-scan at I month shows decrease in the size of granuloma $(4.50 \times I I \times 3.5 \mathrm{~mm})$, with clearing of the central anechoic space.

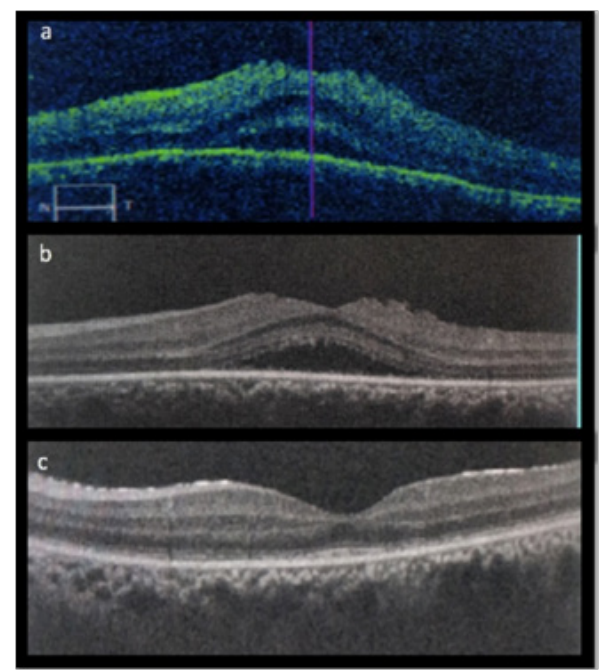

Figure 3 (A) OCT at first visit shows foveal subretinal fluid with slightly convex RPE elevation. (B) shows persistent SRF at macula at $3^{\text {rd }}$ month, c) OCT showing resolution of SRF after intravitreal triamcinolone acetonide injection.

\section{Discussion}

TB is caused by Mycobacterium tuberculosis, commonly described as a systemic disease that involves not only the lungs, but many other organs, including the eye. Ocular TB is a rare( $1 \%$ of all cases of TB), can involve any part of eye and can occur with or without evidence of systemic TB. Large solitary granuloma is a common presentation and are present as a solitary yellowish or grayish white large lesion, generally located in the posterior pole. ${ }^{1}$ Diagnosis of TB requires microbiological confirmation and as this can be challenging in ocular tissue or fluids, evidence of systemic tuberculosis not only contributes to the diagnosis of presumed ocular tuberculosis but also provides specimens for microbiological and histopathological confirmation. ${ }^{2}$ Ultrasonography in granuloma shows low to moderate internal reflectivity, making it difficult to distinguish them from amelanotic malignant melanoma. The presence of an anechoic space in the mass points toward an inflammatory etiology. ${ }^{3}$ A thorough systemic evaluation, including radiographic studies is needed to detect other involved sites. Visual prognosis may be guarded but visual recovery in choroidal tuberculoma depends on involution to a flat inactive scar and can occur with proper and rapid diagnosis with early initiation of treatment depending on the location and size of the tuberculomas. ${ }^{3}$ Systemic treatment with the first-line combination regimen comprising isoniazid, rifampin, pyrazinamide, and ethambutol for a total of 6-12 months has been accepted as standard therapy. ${ }^{4}$ In the present case, a favorable response to treatment was demonstrated by the shrinking of the tuberculoma with oral ATT and steroid therapy, but visual gain was documented after treating with triamcinolone acetonide which resulted in resolution of the SRF and shrinkage of the granuloma. This case highlights a rare clinical scenario wherein choroidal tuberculoma is the presenting sign of TB. Ophthalmologists should be aware that solitary choroidal tuberculoma can occur in immunocompetent individuals. It is believed that ultrasonography is an easy and practical method for not only suspecting the diagnosis but also monitoring disease evolution and response to treatment. This is the first case which highlights the use of intravitreal triamcinolone acetonide in management of choroidal tuberculoma.

\section{Acknowledgments}

None.

\section{Conflicts of interest}

No proprietary interest or conflict.

\section{Funding}

No financial funding disclosure.

\section{References}

1. Arej N, Fadlallah A, Chelala E. Choroidal tuberculoma as a presenting sign of tuberculosis. Int Med Case Rep J. 2016;9:365-368.

2. Bodaghi B, LeHoang P. Ocular tuberculosis. Current opinion in ophthalmology. 2000;11(6):443-448

3. Levecq LJ, De Potter P. Solitary choroidal tuberculoma in an immunocompetent patient. Arch Ophthalmol. 2005;123(6):864-866.

4. Zhang M, Zhang J, Liu Y. Clinical presentations and therapeutic effect of presumed choroidal tuberculosis. Retina. 2012;32(4):805-813. 